ARTICLE

DOI: $10.1038 / s 41467-017-00749-4$

OPEN

\title{
Ultra-strong nonlinear optical processes and trigonal warping in $\mathrm{MoS}_{2}$ layers
}

\author{
Antti Säynätjoki ${ }^{1,2}$, Lasse Karvonen ${ }^{1}$, Habib Rostami ${ }^{3}$, Anton Autere (i) 1, Soroush Mehravar ${ }^{4}$, \\ Antonio Lombardo ${ }^{5}$, Robert A. Norwood ${ }^{4}$, Tawfique Hasan ${ }^{5}$, Nasser Peyghambarian 1,2,4, Harri Lipsanen (1) ${ }^{1}$, \\ Khanh Kieu ${ }^{4}$, Andrea C. Ferrari ${ }^{5}$, Marco Polini ${ }^{3} \&$ Zhipei Sun ${ }^{1}$
}

Nonlinear optical processes, such as harmonic generation, are of great interest for various applications, e.g., microscopy, therapy, and frequency conversion. However, high-order harmonic conversion is typically much less efficient than low-order, due to the weak intrinsic response of the higher-order nonlinear processes. Here we report ultra-strong optical nonlinearities in monolayer $\mathrm{MoS}_{2}\left(1 \mathrm{~L}-\mathrm{MoS}_{2}\right)$ : the third harmonic is 30 times stronger than the second, and the fourth is comparable to the second. The third harmonic generation efficiency for $1 \mathrm{~L}-\mathrm{MoS}_{2}$ is approximately three times higher than that for graphene, which was reported to have a large $\chi^{(3)}$. We explain this by calculating the nonlinear response functions of $1 \mathrm{~L}$ $\mathrm{MoS}_{2}$ with a continuum-model Hamiltonian and quantum mechanical diagrammatic perturbation theory, highlighting the role of trigonal warping. A similar effect is expected in all other transition-metal dichalcogenides. Our results pave the way for efficient harmonic generation based on layered materials for applications such as microscopy and imaging.

\footnotetext{
${ }^{1}$ Department of Electronics and Nanoengineering, Aalto University, Tietotie 3, Fl-02150 Espoo, Finland. ${ }^{2}$ Institute of Photonics, University of Eastern Finland, Yliopistokatu 7, Fl-80100 Joensuu, Finland. ${ }^{3}$ Istituto Italiano di Tecnologia, Graphene Labs, Via Morego 30, I-16163 Genova, Italy. ${ }^{4}$ College of Optical Sciences, University of Arizona, 1630 E University Blvd, Tucson, AZ 85721, USA. ${ }^{5}$ Cambridge Graphene Centre, University of Cambridge, Cambridge CB3 OFA, UK. Antti Säynätjoki, Lasse Karvonen and Habib Rostami contributed equally to this work. Correspondence and requests for materials should be addressed to Z.S. (email: zhipei.sun@aalto.fi)
} 
$\mathrm{N}$ onlinear optical phenomena can generate high-energy photons by converting $n=2,3,4, \ldots$ low-energy photons into one high-energy photon. These are usually referred to as second-, third-, and fourth-harmonic generation (SHG, THG, and $\mathrm{FHG})^{1}$. Due to different selection rules ${ }^{1,2}$, harmonic processes are distinct from optically pumped laser phenomena (e.g., optically pumped amplification ${ }^{3}$ ), and other typical single-photon processes (e.g., single-photon excited photoluminescence ${ }^{1}$ ), in which the energy of the generated photons is smaller than the pump photons. Multiphoton harmonic processes have been widely exploited for various applications (e.g., all-optical signal processing in telecommunications $^{1,4}$, medicine $e^{5}$, and data storage ${ }^{6}$ ), as well as to study various transitions forbidden under low-energy single-photon excitation $^{5,6}$. The physical origin of these processes is the nonlinear polarization induced by an electromagnetic field $\mathbf{E}$. This gives rise to higher harmonic components, the $n$-th harmonic component amplitude being proportional ${ }^{1}$ to $|\mathbf{E}|^{n}$. Quantum mechanically, higher-harmonic generation involves the annihilation of $n$ pump photons and generation of a photon with $n$ times the pump energy. Because an $n$-th order nonlinear optical process requires $n$ photons to be present simultaneously, the probability of higher-order processes is lower than that of lower order ${ }^{1}$. Thus, higher-order processes are typically weaker and require higher pump intensities 7,8 .

Graphene and related materials are at the center of an everincreasing research effort due to their unique and complementary properties, making them appealing for a wide range of photonic and optoelectronic applications ${ }^{9-11}$. Among these, semiconducting transition-metal dichalcogenides (TMDs) are of particular interest due to their direct bandgap when in monolayer (1L) form ${ }^{12}$, leading to an increase in luminescence by a few orders of magnitude compared with the bulk material ${ }^{12,13} \cdot 1 \mathrm{~L}-\mathrm{MoS}_{2}$ has a single layer of Mo atoms sandwiched between two layers of $S$ atoms in a trigonal prismatic lattice. Therefore, in contrast to graphene, it is non-centrosymmetric and belongs to the space group $D_{3 \mathrm{~h}}^{114}$. The lack of spatial inversion symmetry makes $1 \mathrm{~L}-\mathrm{MoS}_{2}$ an interesting material for nonlinear optics, since second-order nonlinear processes are present only in non-centrosymmetric materials ${ }^{1}$. However, when stacked, $\mathrm{MoS}_{2}$ layers are arranged mirrored with respect to one another ${ }^{14}$, therefore $\mathrm{MoS}_{2}$ with an even number of layers (EN) is centrosymmetric and belongs to the $D_{3 \mathrm{~d}}^{3}$ space group ${ }^{14}$, producing no second harmonic $(\mathrm{SH})$ signal. On the other hand, $\mathrm{MoS}_{2}$ with odd number of layers $(\mathrm{ON})$ is non-centrosymmetric. SHG from 1L$\mathrm{MoS}_{2}$ was reported by several groups ${ }^{14-21}$.

Here we present combined experimental and theoretical work on nonlinear harmonic generation in $1 \mathrm{~L}$ and few-layer (FL) $\mathrm{MoS}_{2}$. We report strong THG and FHG from 1L-MoS 2 . THG is more than one order of magnitude larger than SHG, while FHG has the same magnitude as SHG. This is surprising, since one normally expects the intensity of nonlinear optical processes to decrease with $n^{1,2}$, with the SHG intensity much larger than that in THG and FHG, although even-order processes only exist in non-centrosymmetric materials. Our results show that this expectation is wrong in the case of $1 \mathrm{~L}-\mathrm{MoS}_{2}$. At sufficiently low photon frequencies (in our experiments the photon energy of the pump is $0.8 \mathrm{eV}$ ), SHG only probes the low-energy band structure of 1L$\mathrm{MoS}_{2}$. This is nearly rotationally invariant ${ }^{22-29}$, but with corrections due to trigonal warping. It is because of these corrections $^{23,26,27}$, fully compatible with the $D_{3 \mathrm{~h}}^{1}$ space group ${ }^{1}$, but reducing the full rotational symmetry of the low-energy bands to a three-fold rotational symmetry ${ }^{1}$, that a finite amplitude of nonlinear harmonic processes can exist at low photon energies in EN-MoS 2 . The lack of spatial inversion symmetry is a necessary but not sufficient condition for the occurrence of SHG. A purely isotropic band structure gives a vanishing SHG signal ${ }^{30-33}$, despite some terms in the Hamiltonian explicitly breaking inversion symmetry ${ }^{27}, 34-37$. Terms proportional to the $\sigma_{z}$ Pauli matrix break inversion symmetry. Breaking the continuous rotational symmetry of isotropic models (e.g., by including trigonal warping) is required to obtain a non-zero second-order response in a twoband system. In hexagonal lattices, trigonal warping is a deviation from purely isotropic bands that emerges as one moves away from the corners $\mathbf{K}$ and $\mathbf{K}^{\prime}$ of the Brillouin zone ${ }^{23,26,27,36-38}$. Since the lattice has a honeycomb structure, this distortion displays a threefold rotational symmetry ${ }^{23,26,27,36-38}$. We demonstrate that the observed THG/SHG intensity ratio can be explained by quantum mechanical calculations based on finite-temperature many-body diagrammatic perturbation theory ${ }^{39}$ and low-energy continuummodel Hamiltonians that include trigonal warping ${ }^{35}$. We conclude that, similar to $\mathrm{SHG}^{14-18}$, the THG process is sensitive to the number of layers, their symmetry, relative orientation, as well as a

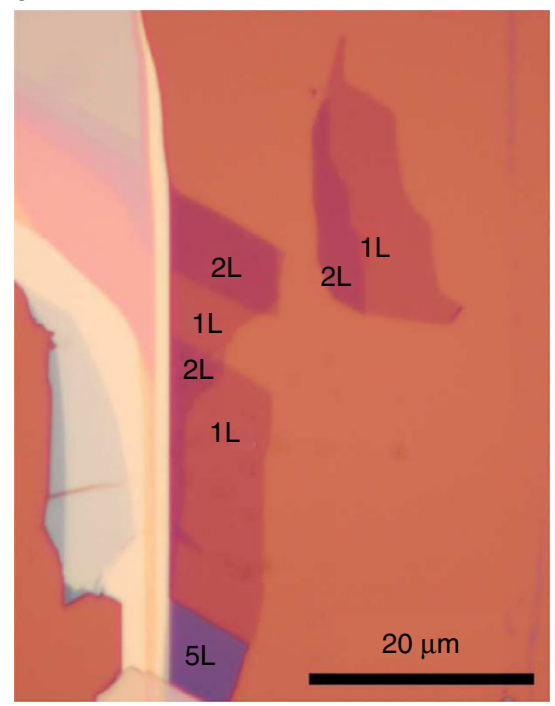

b

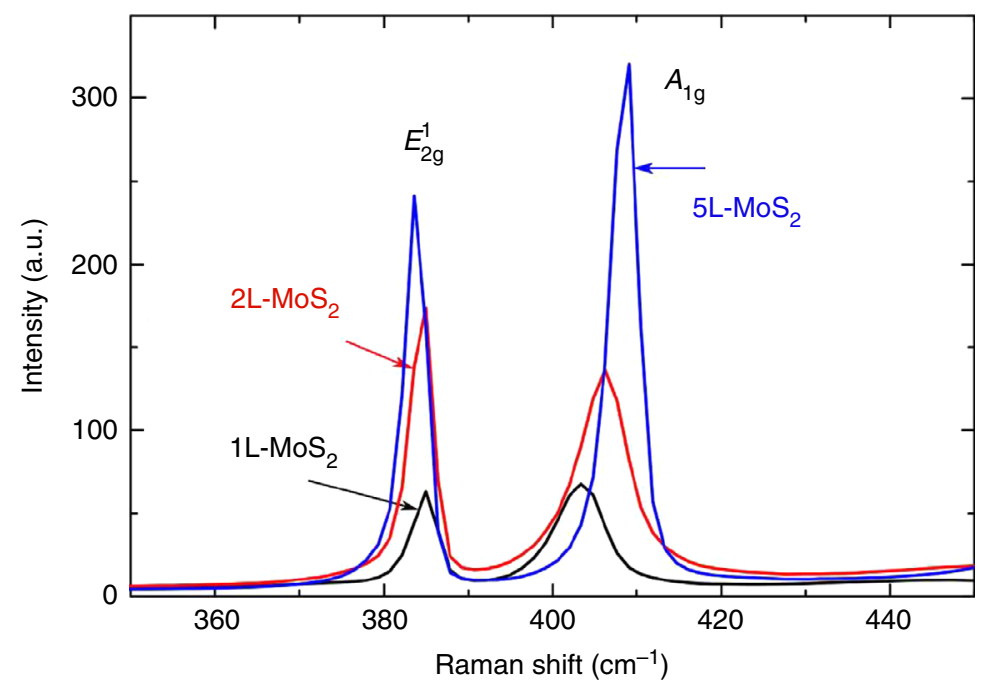

Fig. 1 Optical image and Raman spectra of the $\mathrm{MoS}_{2}$ flakes. a Optical micrograph with single-layer, bilayer, and five-layer areas marked by $1 \mathrm{~L}, 2 \mathrm{~L}$, and $5 \mathrm{~L}$. b Raman spectra of the same sample 




Fig. 2 Schematic diagram of multiphoton microscope. MLL, linearly polarized mode-locked fiber laser. VA, variable attenuator. QWP, quarterwave plate used to study the dependence of SHG and THG on the elliptical polarization of the pump light. BP filter, bandpass filter. PMT, photomultiplier tube

the elliptical polarization of the excitation light. Similar effects are expected for all other TMDs. This paves the way for the assembly of heterostructures with tailored nonlinear optical properties.

\section{Results}

Samples. $\mathrm{MoS}_{2}$ flakes are produced by micromechanical cleavage (MC) of bulk $\mathrm{MoS}_{2}{ }^{40}$ onto $\mathrm{Si}+285 \mathrm{~nm} \mathrm{SiO} 2.1 \mathrm{~L}-\mathrm{MoS}_{2}$ and bilayer $\left(2 \mathrm{~L}-\mathrm{MoS}_{2}\right)$ flakes are identified by a combination of optical contrast $^{41}, 42$ and Raman spectroscopy ${ }^{43}$. Raman spectra are acquired by a Renishaw micro-Raman spectrometer equipped with a 600 line/mm grating and coupled with an $\mathrm{Ar}^{+}$ion laser at $514.5 \mathrm{~nm}$. Figure 1a shows the $\mathrm{MoS}_{2}$ flakes studied in this work and their Raman signatures. A reference MC graphene sample is also prepared and placed on a similar substrate.

SHG and THG charcterization. Nonlinear optical measurements are carried out with the set-up shown in Fig. $2^{44,45}$. As excitation source, we use an erbium-doped mode-locked fiber laser with a $50 \mathrm{MHz}$ repetition rate, maximum average power $60 \mathrm{~mW}$, and pulse duration $150 \mathrm{fs}$, which yields an estimated pulse peak power of $\sim 8 \mathrm{~kW}^{46}$. The laser beam is scanned with a galvo mirror and focused on the sample using a microscope objective. The back-scattered second and third harmonic signals are split into different branches using a dichroic mirror and then detected using photomultiplier tubes (PMTs). For two-channel detection, the light is split into two PMTs using a dichroic mirror with $562 \mathrm{~nm}$ cutoff. After the dichroic mirror, the detected wavelength range can be further refined using bandpass filters. The light can also be directed to a spectrometer (OceanOptics QE Pro-FL). The average power on the sample is kept between 10 and $28 \mathrm{~mW}$ with a typical measurement time $\sim 5 \mu \mathrm{s}$, which prevents sample damage a
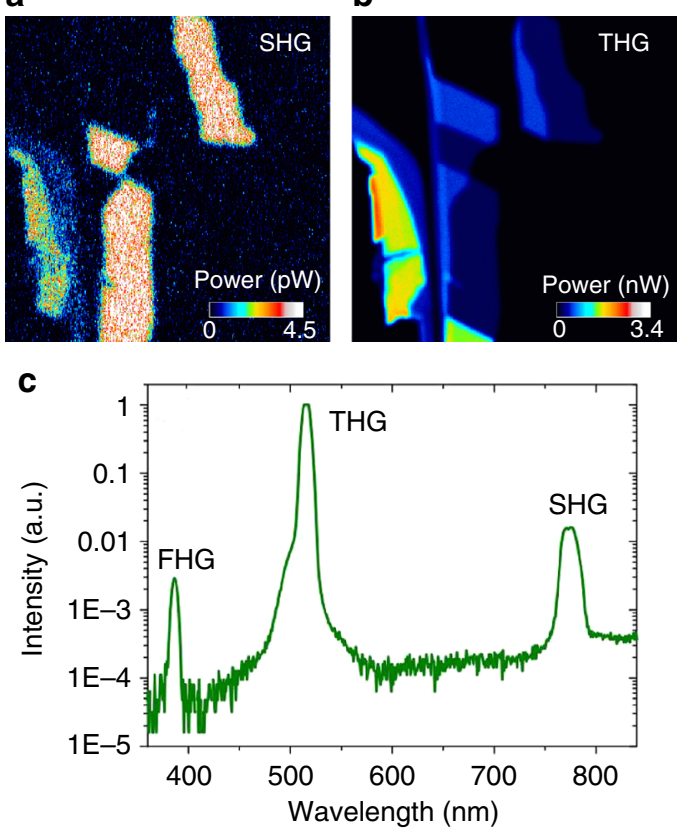

Fig. 3 Multiphoton images of $\mathrm{MoS}_{2}$ flakes. a SHG and $\mathbf{b}$ THG map of the flakes in Fig. 1a. c Optical spectrum of the nonlinear signal from 1L-MoS 2 with a peak irradiance $\sim 30 \mathrm{GW} \mathrm{cm}^{-2}$

and enables high signal-to-noise-ratio, even with acquisition time per pixel in the $\mu$ s range.

SHG and THG images of the $\mathrm{MoS}_{2}$ sample are shown in Fig. 3a, b. The $\mathrm{SH}$ photon energy is $\sim 1.6 \mathrm{eV}$, lower than the bandgap of $1 \mathrm{~L}-\mathrm{MoS}_{2}{ }^{12,13}$. This is not unexpected, as harmonic generation can occur when the harmonic energy is below the bandgap ${ }^{1,47,48}$. The SHG signal is generated in $1 \mathrm{~L}-\mathrm{MoS}_{2}$, while $2 \mathrm{~L}-\mathrm{MoS}_{2}$ appears dark. As discussed above, the secondorder nonlinear response is present in $1 \mathrm{~L}-\mathrm{MoS}_{2}$, which is noncentrosymmetric. However, when stacked to form $2 \mathrm{~L}-\mathrm{MoS}_{2}$, $\mathrm{MoS}_{2}$ layers are mirrored ${ }^{14}, 15$. Therefore, $\mathrm{EN}-\mathrm{MoS}_{2}$ is centrosymmetric $^{14,15}$, and belongs to the $D_{3 \mathrm{~d}}^{3}$ space group ${ }^{14,15}$, producing no SHG signal. On the other hand, $\mathrm{ON}-\mathrm{MoS}_{2}$ flakes are non-centrosymmetric ${ }^{14}, 15$.

We note that strong THG is detected compared with SHG, even for $1 \mathrm{~L}-\mathrm{MoS}_{2}$, Fig. 3b. THG was previously reported for a 7L-MoS flake ${ }^{18}$, but here we see it down to $1 \mathrm{~L}-\mathrm{MoS}_{2}$. Reference ${ }^{49}$ followed our work ${ }^{50}$ and reported THG and SHG from $1 \mathrm{~L}-\mathrm{MoS}_{2}$, giving effective bulk-like second- and third-order susceptibilities $\chi_{\text {eff }}^{(2)}$ and $\chi_{\text {eff }}^{(3)}$ of $2.9 \times 10^{-11} \mathrm{mV}^{-1}$ and $2.4 \times 10^{-19} \mathrm{~m}^{2} \mathrm{~V}^{-2}$, respectively. However, ref. ${ }^{49}$ did not provide a detailed explanation of the large THG signal compared to the SHG. Instead it assigned the large THG/SHG ratio to a possible enhancement of THG by the edge of the $\mathrm{B}$ exciton. However, refs. ${ }^{1}, 52$ demonstrated that $\mathrm{SHG}$ is enhanced only when the SHG wavelength overlaps the A or B excitons. A similar behavior is expected for THG. Thus, the explanation in ref. ${ }^{49}$ may not be correct. Reference ${ }^{53}$ reported high-harmonic (>6th-order) generation in the non-perturbative regime with mid-infrared (IR) excitation $(0.3 \mathrm{eV})$, unlike our THG and FHG results with near-IR excitation $(0.8 \mathrm{eV})$. We do not detect THG from the thickest areas of our flake, with $N>30$, as in ref. ${ }^{18}$. The output spectrum in Fig. $3 c$ further confirms that we observe both SHG and THG. Peaks for THG and SHG at 520 and $780 \mathrm{~nm}$ can be seen, as well as at $390 \mathrm{~nm}$, corresponding to a four-photon process. This is detected only in $1 \mathrm{~L}-\mathrm{MoS}_{2}$. Its intensity is $\sim 5.5$ times lower than SHG, and two orders of magnitude smaller than THG. 

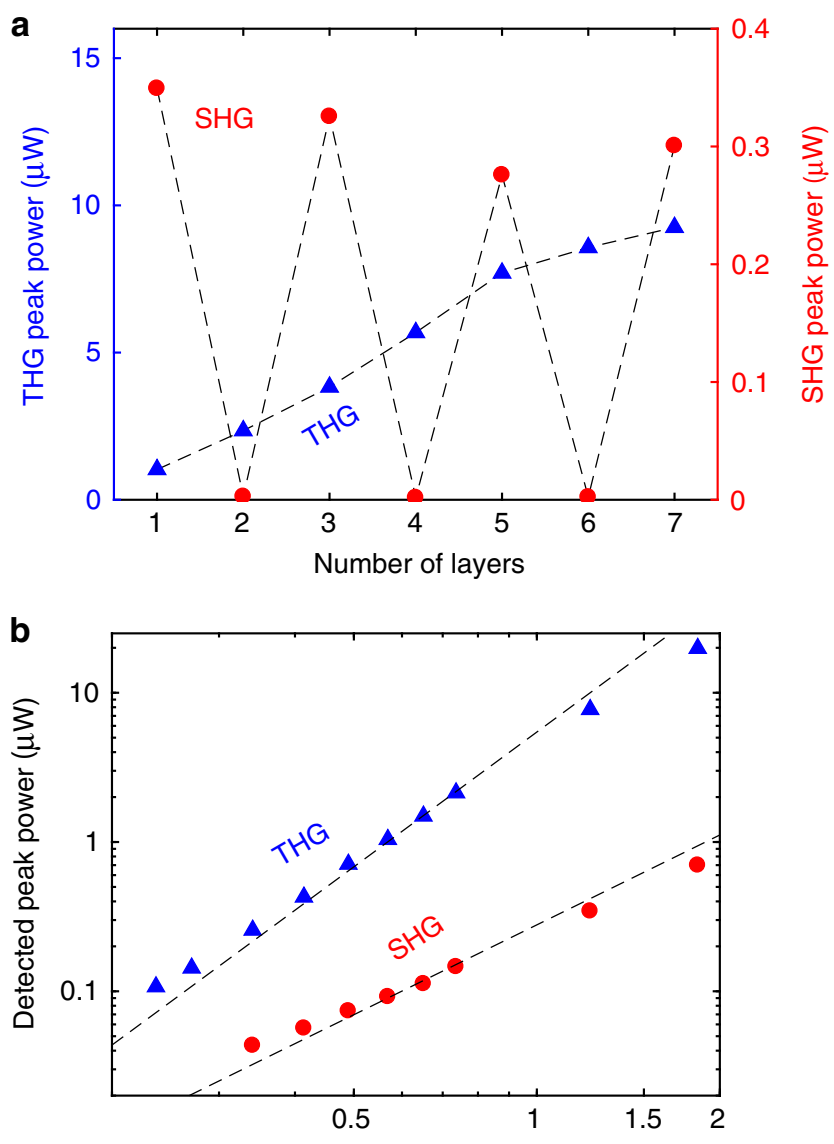

Pump peak power on sample (kW)

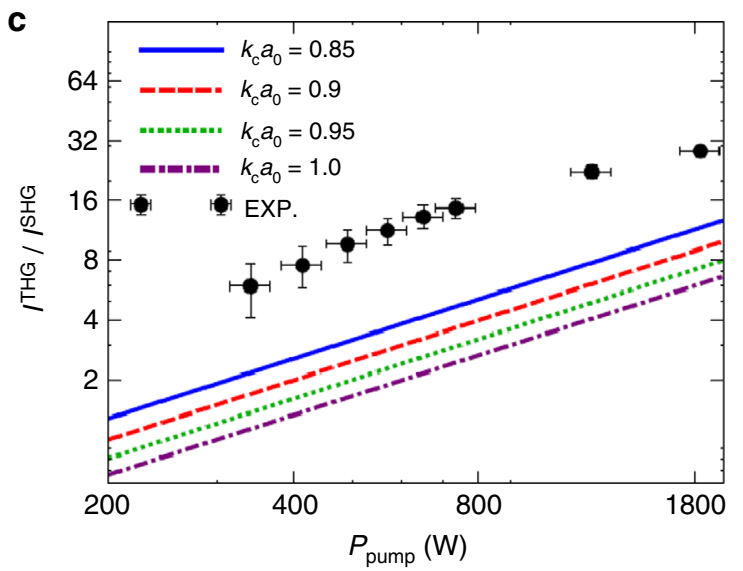

Fig. 4 Experimental and theoretical nonlinear optical processes in $\mathrm{MoS}_{2}$. a $\mathrm{SHG}$ and THG intensities as functions of $\mathrm{N}$. $\mathbf{b}$ Power dependence of SHG and THG in $1 \mathrm{~L}-\mathrm{MoS}_{2}$. c Experimental and theoretical THG/SHG irradiance ratio as a function of $P_{\text {pump. }}$. Different theoretical curves refer to different values of the ultra-violet cutoff $k_{\mathrm{c}}$ (in units of $1 / a_{0}=\sqrt{3} / a$, where $a=3.16 \AA$ is the lattice constant of $1 \mathrm{~L}-\mathrm{MoS}_{2}{ }^{12}$ ). Black dashed lines in panels $\mathbf{a}$ and $\mathbf{b}$ are a guide to the eye. The error bars in $\mathbf{c}$ account for experimental uncertainties

SHG signals on areas with $N=3,5,7$ have nearly the same intensity as $1 \mathrm{~L}-\mathrm{MoS}_{2}$, Fig. 4 a. This contrasts ref. ${ }^{14}$, where a pump laser at $810 \mathrm{~nm}$ was used. We attribute this difference to the fact that photons generated in the second-order nonlinear process in our set-up with a $1560 \mathrm{~nm}$ pump have an energy $\sim 1.6 \mathrm{eV}$
$(780 \mathrm{~nm})$, below the band gap of $1 \mathrm{~L}-\mathrm{MoS}_{2}{ }^{12}$, therefore are not adsorbed, unlike the SHG signal in ref. ${ }^{14}$.

Second- and third-order nonlinear susceptibilities. Based on the measured SHG and THG intensities, we can estimate the nonlinear susceptibilities $\chi^{(2)}$ and $\chi^{(3)} \cdot \chi^{(2)}$ can be calculated from the measured average powers of the fundamental and $\mathrm{SH}$ signals as follows ${ }^{54}$ :

$$
\chi_{\mathrm{s}}^{(2)}=\sqrt{\frac{\epsilon_{0} c \lambda_{2}^{4} P_{2 \omega} R \tau^{2}\left(n_{2}+1\right)^{2}\left(n_{1}+1\right)^{2}}{32 N_{a}^{2} \tau_{2} P_{\text {pump }} \phi}},
$$

where $\tau$ is the pulse width, $P_{\text {pump }}$ is the average power of the incident fundamental (pump) beam, and $P_{2 \omega}$ stands for the generated $\mathrm{SH}$ beam power, $R$ is the repetition rate, $N_{a}=0.5$ is the numerical aperture, $\lambda_{2}=780 \mathrm{~nm}$ is the $\mathrm{SH}$ wavelength, $\tau=\tau_{2}=150 \mathrm{fs}$ are the pulse durations at fundamental and SH wavelengths, $\quad \phi=8 \pi \int_{0}^{1}\left|\cos ^{-1} \rho-\rho \sqrt{1-\rho^{2}}\right|^{2} \rho \mathrm{d} \rho=3.56$ from ref. ${ }^{54}$, and $n_{1}=n_{2} \sim 1.45$ are the refractive indexes of the substrate at the wavelengths of the fundamental and SHG, respectively. The effective bulk-like second-order susceptibility of $\operatorname{MoS}_{2}\left(\chi_{\text {eff }}^{(2)}\right)$ can be obtained from Eq. (1) with $\chi_{\text {eff }}^{(2)}=\frac{\chi_{\mathrm{s}}^{(2)}}{t_{\mathrm{Mos}_{2}}}$, where $t_{\mathrm{MoS}_{2}}=0.65 \mathrm{~nm}$ is the $1 \mathrm{~L}-\mathrm{MoS}_{2}$ thickness ${ }^{10,24}$. We obtain the effective second-order susceptibility $\chi_{\text {eff }}^{(2)} \sim 2.2 \mathrm{pmV}^{-1}$ for $1 \mathrm{~L}$ $\mathrm{MoS}_{2}$. Reference ${ }^{49}$ reported a bulk-like second-order susceptibility $29 \mathrm{pmV}^{-1}$, which is $\sim 10$ times larger than here. However, several other studies reported $\sim 5 \mathrm{pmV}^{-1}$ for $1560 \mathrm{~nm}^{20}, 52,55$. Thus, our measured $\chi_{\text {eff }}^{(2)}$ agrees well with earlier values measured with similar excitation wavelength.

The third-order susceptibility $\chi_{\text {eff }}^{(3)}$ can be estimated by comparing the measured THG signal from $\mathrm{MoS}_{2}$ to that of 1L-graphene (SLG):

$$
\chi_{\mathrm{eff}}^{(3)} \approx \frac{t_{\mathrm{SLG}}}{t_{\mathrm{MoS}_{2}}} \sqrt{\frac{\mathrm{THG}_{\mathrm{MoS}_{2}}}{\mathrm{THG}_{\mathrm{SLG}}}} \chi_{\mathrm{SLG}}^{(3)} .
$$

with $t_{\mathrm{SLG}}=0.33 \mathrm{~nm}$ the SLG thickness, and $\mathrm{THG}_{\mathrm{SLG}}$ and $\mathrm{THG}_{\mathrm{MoS}_{2}}$ the measured signals from SLG and $\mathrm{MoS}_{2}$, respectively. Our results show that THG from $1 \mathrm{~L}-\mathrm{MoS}_{2}$ is around three times larger than $\mathrm{THG}_{\mathrm{SLG}}$, which indicates that $\chi^{(3)}$ of $1 \mathrm{~L}-\mathrm{MoS}_{2}$ is comparable to that of SLG, in the frequency range of our experiments. Previous reports indicate 49,56 that $\chi_{\mathrm{SLG}}^{(3)}$ is $\sim 10^{-17}-10^{-19} \mathrm{~m}^{2} \mathrm{~V}^{-2}$. Thus, based on Eq. (2), $\chi^{(3)}$ of $1 \mathrm{~L}-\mathrm{MoS}_{2}$ is in the same range. This is remarkable, as SLG is known to have a large $\chi^{(3) 56-59}$. Reference ${ }^{18}$ reported $\chi^{(3)}$ of $7 \mathrm{~L}-\mathrm{MoS}_{2}$ to be approximately three orders of magnitude smaller than $\chi_{\text {SLG }}^{(3)}$ of ref. ${ }^{56} \cdot \chi_{\text {SLG }}^{(3)}$ from ref. ${ }^{56}$ is much higher than other theoretical ${ }^{58}$ and experimental ${ }^{49}$ values. We believe that our measured ratio between $1 \mathrm{~L}-\mathrm{MoS}_{2}$ and SLG is more accurate, since we measured both materials at the same time under the same conditions.

We note that large discrepancies can be found in earlier reported effective susceptibilities for layered materials (LM). For example, there is a approximately four orders of magnitude difference in $\chi^{(3)}$ for SLG $\left(\sim 10^{-15} \mathrm{~m}^{2} \mathrm{~V}^{-2}\right.$ in ref. ${ }^{57} ; \sim 10^{-19} \mathrm{~m}^{2} \mathrm{~V}^{-2}$ in ref. $\left.{ }^{49}\right)$. There is an approximately three orders of magnitude difference in $\chi^{(2)}$ reported for $1 \mathrm{~L}-\mathrm{MoS}_{2}$ at $800 \mathrm{~nm}$ (e.g., $\sim 10^{-7} \mathrm{mV}^{-1}$ in ref. ${ }^{15}$; and $\sim 10^{-10} \mathrm{mV}^{-1}$ in ref. ${ }^{17}$ ). Effective susceptibilities are well defined only in three-dimensional materials, since their definition involves a polarization per unit volume ${ }^{1}$. Therefore, given the large discrepancies in literature, it is better to describe the nonlinear processes in LMs using the ratio between the harmonic signal power and the incident pump power (i.e., harmonic conversion efficiency). In this case, when comparing the efficiencies in our measurements with those in ref. ${ }^{49}$, our THG efficiency $\left(\sim 4.76 \times 10^{-10}\right)$ is $\sim 1.4$ times 


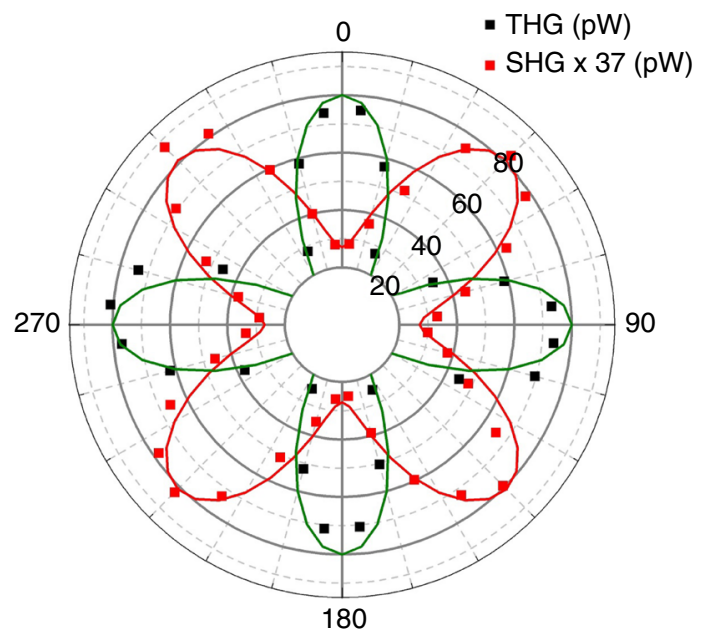

Fig. $5 \mathrm{SHG}$ and THG dependence on the pump light polarization. The polar plot angle corresponds to linearly polarized light when $\theta=0^{\circ}+m \cdot 90^{\circ}$, and to circularly polarized pump light when $\theta=45^{\circ}+m \cdot 90^{\circ}$. The SHG power is multiplied by a factor of 37 to fit in the same scale as THG

larger than that $\left(\sim 3.38 \times 10^{-10}\right)$ in ref. ${ }^{49}$, while our SHG efficiency $\left(\sim 6.47 \times 10^{-11}\right)$ is twice that of ref. ${ }^{49}$. Since the effective susceptibilities are not well defined for LMs and also depend on the calculation method, we believe that the conversion efficiency is a better figure of merit for LMs.

\section{Discussion}

Our measurements show that the nonlinear response of $1 \mathrm{~L}-\mathrm{MoS}_{2}$ and SLG are comparable in magnitude, both revealing stronger nonlinear efficiency than three-dimensional nonlinear materials, such as diamond and quartz ${ }^{59}$. This can be explained by considering their effective Hamiltonians ${ }^{27,34-37,60}$. The main contribution to THG is paramagnetic. This is described by the square diagram in Supplementary Notes 1-4 (Supplementary Figs. 1-6). This paramagnetic contribution is mainly related to the strong inter-band coupling in the effective Hamiltonian, controlled by large velocity scales, $v_{\mathrm{F}} \approx \frac{c}{300}$ and $v=\frac{t_{0} a_{0}}{\hbar} \approx$ $0.65 \times \frac{c}{300}$ for SLG and $1 \mathrm{~L}-\mathrm{MoS}_{2}$, with $c$ the speed of light. The SLG paramagnetic third-harmonic efficiency (PTHE) is proportional to the square of third-order conductivity. Since ${ }^{39}$ $\sigma_{y y y y}^{(3)} \propto v_{\mathrm{F}}^{2}$, we get an overall prefactor $v_{\mathrm{F}}^{4}$, which explains the strong nonlinear SLG response. Similarly, for $1 \mathrm{~L}-\mathrm{MoS}_{2}$, the square diagram contains four paramagnetic current vertices, which gives an overall prefactor $v^{4}$, and an integral over the dummy momentum variables, which gives a prefactor $\frac{1}{v^{2}}$ (see Supplementary Note 3). Therefore, the third-order response function, $\Pi_{y y y y}^{(3)}$, is proportional to $v^{2}$, which implies a scaling of PTHE as $v^{4}$. Exciton physics is not considered because our experimental conditions only capture off-resonance transitions.

$1 \mathrm{~L}-\mathrm{MoS}_{2}$ is transparent at this wavelength due to its $\sim 1.9 \mathrm{eV}$ gap $^{12}$, while SLG absorbs $2.3 \%$ of the light ${ }^{61}$. Therefore, $1 \mathrm{~L}-\mathrm{MoS}_{2}$ and other TMDs are promising for integration with waveguides or fibers for all-optical nonlinear devices, such as all-optical modulators and signal processing devices, where materials with nonlinear properties are essential ${ }^{11}$.

The SHG and THG power dependence follows quadratic and cubic trends, Fig. 4b. At our power levels, THG is up to 30 times stronger than SHG. 1L-TMDs have strongly bound excitons that can modify their optical properties ${ }^{62-64}$. The exciton resonances also affect their nonlinear optical responses ${ }^{17,65,66}$. References 51,55 reported that when the SHG energy is above the A and B excitons, resonance effects are not observed. In our experiments, the energy of $3 \omega$ photons is above the A exciton but does not directly overlap with the A or B excitons. Thus, we do not assign the large THG/SHG intensity ratio to an excitonic enhancement, but to the approximate rotational invariance of the $1 \mathrm{~L}-\mathrm{MoS}_{2}$ band structure at low energies, which is broken by trigonal warping.

SHG is weaker than expected for a non-centrosymmetric material, due to near-isotropic bands contributing to the SHG signal for our low incident photon energies $(0.8 \mathrm{eV})$. Even in the presence of a weak trigonal warping, SHG and THG might be comparable above the threshold for two- and three-photon absorption edges. However, this is not a resonant effect. Resonances only emerge when the laser matches a single level (like an excitonic level) rather than a continuum of states ${ }^{67}$. In our analysis, SHG would be absent without trigonal warping. But, trigonal warping alone cannot explain the magnitude of the FHG signal compared to SHG and THG.

Figure $4 \mathrm{c}$ compares the THG/SHG ratio from experiments and calculations based on the $\boldsymbol{k} \cdot \boldsymbol{p}$ theory ${ }^{35}$ (see Supplementary Note 1) and finite-temperature diagrammatic perturbation theory $^{39}$ (see Supplementary Notes 3 and 4). The calculations are a factor 2 smaller than the experiments. Considering the complexity of the nonlinear optical processes and that our calculations ignore high-energy band structure effects ${ }^{29}$ and many-body renormalizations ${ }^{65}$, we believe this to be a satisfactory agreement, indicating the importance of trigonal warping in harmonic generation.

FHG generally derives from cascades of lower-order nonlinear multiphoton processes ${ }^{68}$. With an excitation wavelength of $1560 \mathrm{~nm}$, this could be, e.g., a cascade of two SHG processes, where $780 \mathrm{~nm}$ photons are first generated through SHG $\left(\omega_{1560 \mathrm{~nm}}+\omega_{1560 \mathrm{~nm}} \Rightarrow \omega_{780 \mathrm{~nm}}\right)$ and then undergo another SHG process $\left(\omega_{780 \mathrm{~nm}}+\omega_{780 \mathrm{~nm}} \Rightarrow \omega_{390 \mathrm{~nm}}\right)$. To yield a FHG at $390 \mathrm{~nm}$ of the same intensity as SHG at $780 \mathrm{~nm}$ in this cascaded process, one would need a conversion efficiency (defined as $P_{2 \omega} / P_{\text {pump }}{ }^{1}$ ) for the second SHG process (i.e., $\omega_{780 \mathrm{~nm}}+\omega_{780 \mathrm{~nm}} \Rightarrow \omega_{390 \mathrm{~nm}}$ ) to be close to unity. However, we observe a conversion efficiency $\sim 10^{-10}$ for SHG. Therefore, we conclude that our FHG does not arise from cascaded SHGs. Another possible cascade process is based on THG $\left(\omega_{1560 \mathrm{~nm}}+\omega_{1560 \mathrm{~nm}}+\omega_{1560 \mathrm{~nm}} \Rightarrow \omega_{520 \mathrm{~nm}}\right)$ and sum-frequency generation $\left(\omega_{520 \mathrm{~nm}}+\omega_{1560 \mathrm{~nm}} \Rightarrow \omega_{390 \mathrm{~nm}}\right)$. We find that THG strongly increases up to $N=5$, as for Fig. 4a. Therefore, we expect this cascaded process to have a similar trend with $N$. However, we only observe FHG in $1 \mathrm{~L}-\mathrm{MoS}_{2}$. Thus, we also exclude this cascade process, and conclude that this is a direct $\chi^{(4)}$ process.

We now consider the dependence of our results on the elliptical polarization of the incident light. We consider an incident laser beam with arbitrary polarization, i.e., $\mathbf{E}=|\mathbf{E}| \hat{\varepsilon}_{ \pm}$with $\hat{\varepsilon}_{ \pm}=\widehat{\mathbf{x}} \cos (\theta) \pm i \widehat{\mathbf{y}} \sin (\theta)$. Using the crystal symmetries of $1 \mathrm{~L}-\mathrm{MoS}_{2}$, we derive (see Supplementary Note 2) the following expressions for the second- and third-order polarizations $\mathbf{P}^{(2)}$ and $\mathbf{P}^{(3)}$ :

$$
\mathbf{P}^{(2)}=\epsilon_{0} \chi_{y y y}^{(2)}|\mathbf{E}|^{2}[\mp i \sin (2 \theta) \widehat{\mathbf{x}}-\widehat{\mathbf{y}}]
$$

and

$$
\mathbf{P}^{(3)}=\epsilon_{0} \chi_{y y y y}^{(3)}|\mathbf{E}|^{3} \hat{\varepsilon}_{ \pm} \cos (2 \theta) .
$$

Note that $\theta=0^{\circ}$ corresponds to a linearly polarized laser along the $\widehat{\mathbf{x}}$ direction, perpendicular to the $D_{3 \mathrm{~h}}^{1}$ mirror symmetry plane, while $\theta=45^{\circ}$ corresponds to a circularly polarized laser. From Eq. (3), we expect the intensity of SHG in response to a circularly polarized pump laser to be twice that of a linearly polarized laser. 

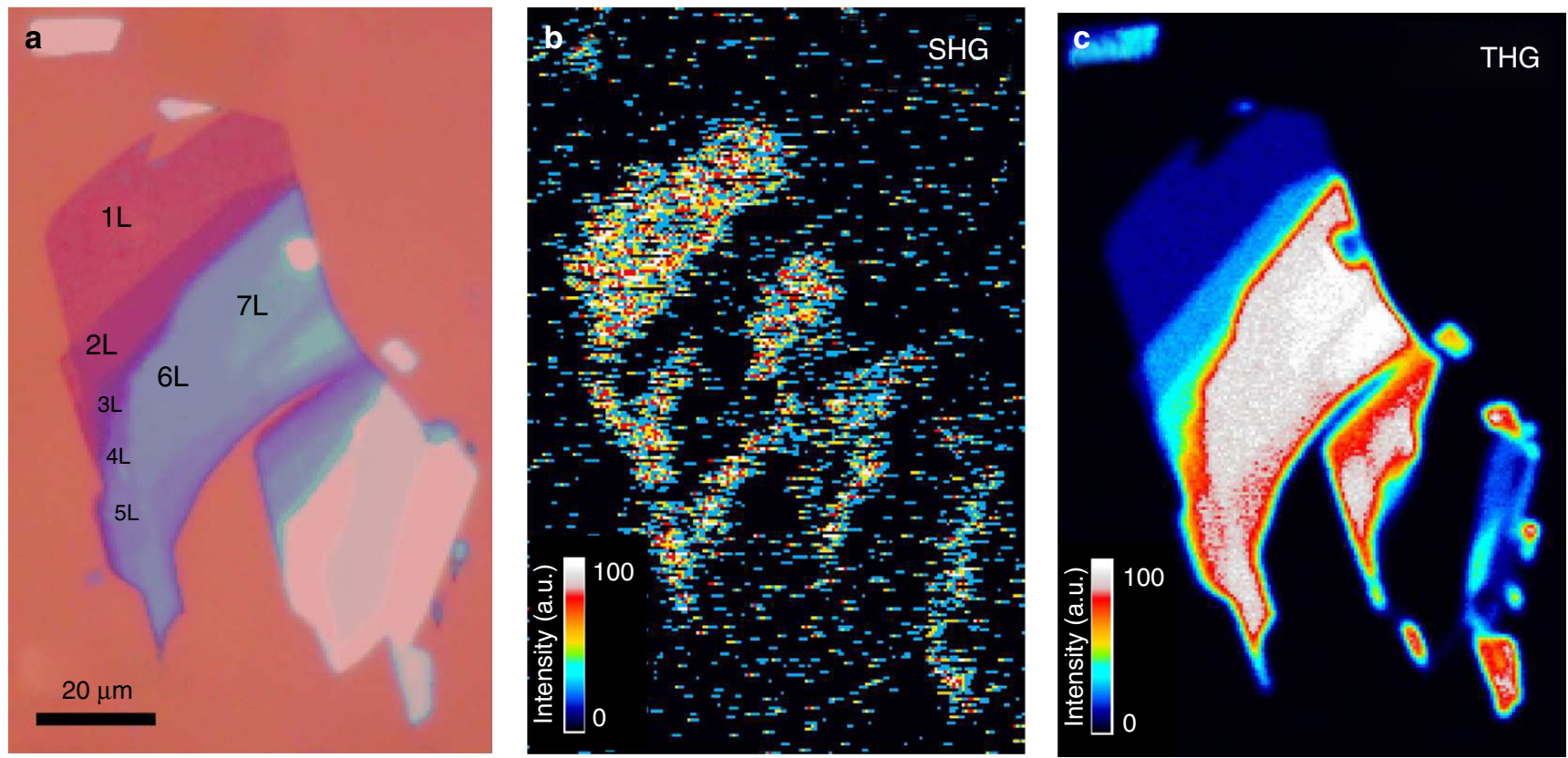

Fig. 6 Optical and multiphoton images of few-layer $\mathrm{MoS}_{2}$ flake. a Optical micrograph, b SHG, and c THG images of flake with few-layer areas under 1560 $\mathrm{nm}$ excitation

Equation (4) implies vanishing THG in response to a circularly polarized pump laser.

We measure the dependence of SHG and THG on elliptical polarization using a linearly polarized laser and a rotating QWP. Depending on the angle $\theta$ between the QWP axes and the laser polarization, the excitation light is linearly $\left(\theta=0^{\circ}+m \cdot 90^{\circ}\right)$ or circularly $\left(\theta=45^{\circ}+m \cdot 90^{\circ}\right)$ polarized. Figure 5 shows that the experimental data are in agreement with Eqs. (3) and (4). The THG signal is maximum for a linearly polarized excitation laser, while it vanishes for circularly polarized light. SHG is always visible, but its intensity is maximum for circularly polarized light.

Given that harmonic generation is strongly dependent on the symmetry and stacking of layers and that different 1L-TMDs (e.g., $\mathrm{WSe}_{2}, \mathrm{MoSe}_{2}$ ) all have similar nonlinear response ${ }^{11,14,15,21}$, one could use heterostructures (e.g., $\mathrm{MoS}_{2} / \mathrm{WSe}_{2}$ ) to engineer SHG and other nonlinear processes for high photon-conversion efficiency for a wide range of applications requiring the generation of higher frequencies. This may lead to the use of LMs and heterostructures for applications utilizing optical nonlinearities (e.g., all-optical devices, frequency combs, high-order harmonic generation, multiphoton microscopy, and therapy etc.).

\section{Methods}

Determination of $\mathbf{M o S}_{2}$ thickness from SHG and THG signals. SHG and THG for FL- $\mathrm{MoS}_{2}(N=1 \ldots 7)$ are studied on the flakes in Fig. 6a. SHG and THG images are shown in Fig. 6b, c. At $1560 \mathrm{~nm}$, the contrast between 1 and $3 \mathrm{~L}$ areas is small, as well as the contrast between 3,5 , and $7 \mathrm{~L}$ regions (Fig. $6 \mathrm{~b}$ ).

The THG signal increases up to $N=7$, Figs. $4 \mathrm{a}$ and $6 \mathrm{c}$. On the other hand, the SHG signal (Fig. 6b) is only generated in ON flakes, due to symmetry ${ }^{14}$. Therefore, areas with intensity between the 3,5, and 7L regions in Fig. 6c, but dark in SHG, are 4 and $6 \mathrm{~L}$. The dependence of the intensities of THG and SHG on $N$ is plotted in Fig. 4a. The combination of SHG and THG can be used to identify $N$ at least up to 7 . The THG signal develops as a function of $N$. Using Maxwell's equations for a nonlinear medium with thickness $t$ and considering the slowly varying amplitude approximation $^{1,69}$, we obtain:

$$
\frac{I_{3 \omega}}{I_{\text {in }}} \approx \frac{(3 \omega)^{2} I_{\text {in }}^{2}}{16 n_{1}^{3} n_{3} \epsilon_{0}^{2} c^{4}}\left|\chi^{(3)}(-3 \omega ; \omega, \omega, \omega)\right|^{2} t^{2} \operatorname{sinc}^{2}\left(\frac{\Delta k t}{2}\right),
$$

where $I_{\text {in }}$ and $I_{3 \omega}$ are the intensities of the incident and THG light, respectively, and $\chi^{(3)}(-3 \omega ; \omega, \omega, \omega)$ is the third-order optical susceptibility, $n_{j=1,3}=\sqrt{\epsilon^{(1)}(j \omega)}$, with $\epsilon^{(1)}$ the TMD linear dielectric function. $\Delta k t$ is the phase mismatch between the fundamental and third harmonic generated waves.

For $\Delta k t \approx 0$, THG adds up quadratically with light propagation length (i.e., $t \propto$ $N)$. The signal starts to saturate for $N=6$. The possible reasons for subquadratic signal build-up can be either phase mismatch, or absorption ${ }^{13}$. For THG, $\Delta k=$ $3 k_{\text {in }} \pm k_{3 \omega}$, where $k_{\text {in }}$ and $k_{3 \omega}$ are the wavevectors of the incident and THG signals, respectively, where the plus sign indicates THG generated in the backward direction, while minus identifies forward generated THG. Even for backward generated THG, $\Delta k t \approx 0$ for $6 \mathrm{~L}-\mathrm{MoS}_{2}\left(\sim 4.3 \mathrm{~nm}^{70}\right)$. This rules out phase mismatch as the origin of the signal saturation when $N \leq 6$. Therefore, we assume that the signal saturation is due to absorption of the third harmonic light.

Diagrammatic nonlinear response theory. To quantify theoretically the strength of nonlinear harmonic generation processes, we generalize the diagrammatic perturbation theory approach ${ }^{39}$ to the case of TMDs. We combine this technique with a low-energy $\boldsymbol{k} \cdot \boldsymbol{p}$ model Hamiltonian $\mathcal{H}(\mathbf{k})$ for $1 \mathrm{~L}-\mathrm{MoS}_{2}{ }^{35}$. In such low-energy model, light-matter interactions are treated by employing minimal coupling ${ }^{35}$, 39 , $\mathbf{k} \rightarrow \mathbf{k}+e \mathbf{A}(t) / \hbar$, where $\mathbf{A}(t)$ is a time-dependent uniform vector potential. Nonlinear response functions are calculated via the multi-legged Feynman diagrams depicted in Supplementary Figs. 2 and 3.

Data availability. The data that support the findings of this study are available from the corresponding author on request.

Received: 8 November 2016 Accepted: 26 July 2017 Published online: 12 October 2017

\section{References}

1. Boyd, R. W. Nonlinear Optics (Academic Press, 2003).

2. Pavone, F. S. \& Campagnola, P. J. Second Harmonic Generation Imaging (Taylor \& Francis, 2013).

3. Saleh, B. E. A. \& Teich, M. C. Fundamentals of Photonics (Academic Press, 2003).

4. Willner, A. E., Khaleghi, S., Chitgarha, M. R. \& Yilmaz, O. F. All-optical signal processing. J. Lightwave Technol 32, 660-680 (2014).

5. Zipfel, W. R., Williams, R. M. \& Webb, W. W. Nonlinear magic: multiphoton microscopy in the biosciences. Nat. Biotechnol. 21, 1369-1377 (2003).

6. Bhawalkar, J. D., He, G. S. \& Prasad, P. N. Nonlinear multiphoton processes in organic and polymeric materials. Rep. Prog. Phys. 59, 1041 (1996). 
7. Tsang, T. Y. F. Optical third-harmonic generation at interfaces. Phys. Rev. A 52, 4116 (1995).

8. Karvonen, L. et al. Investigation of second-and third-harmonic generation in few-layer gallium selenide by multiphoton microscopy. Sci. Rep. 5, 10334 (2015).

9. Bonaccorso, F., Sun, Z., Hasan, T. \& Ferrari, A. C. Graphene photonics and optoelectronics. Nat. Photon 4, 611-622 (2010).

10. Ferrari, A. C. et al. Science and technology roadmap for graphene, related two-dimensional crystals, and hybrid systems. Nanoscale 7, 4598-4810 (2015).

11. Sun, Z., Martinez, A. \& Wang, F. Optical modulators with 2D layered materials. Nat. Photon. 10, 227-238 (2016).

12. Mak, K. F., Lee, C., Hone, J., Shan, J. \& Heinz, T. F. Atomically thin $\mathrm{MoS}_{2}$ : a new direct-gap semiconductor. Phys. Rev. Lett. 105, 136805 (2010).

13. Eda, G. et al. Photoluminescence from chemically exfoliated $\mathrm{MoS}_{2}$. Nano Lett. 11, 5111-5116 (2011).

14. Li, Y. et al. Probing symmetry properties of few-layer $\mathrm{MoS}_{2}$ and h-BN by optical second-harmonic generation. Nano Lett. 13, 3329-3333 (2013).

15. Kumar, N. et al. Second harmonic microscopy of monolayer $\mathrm{MoS}_{2}$. Phys. Rev. B 87, 161403 (2013)

16. Wang, K. et al. Ultrafast saturable absorption of two-dimensional $\mathrm{MoS}_{2}$ nanosheets. ACS Nano 7, 9260-9267 (2013).

17. Malard, L. M., Alencar, T. V., Barboza, A. P. M., Mak, K. F. \& de Paula, A. M. Observation of intense second harmonic generation from $\mathrm{MoS}_{2}$ atomic crystals. Phys. Rev. B 87, 201401 (2013).

18. Wang, R. et al. Third-harmonic generation in ultrathin films of $\mathrm{MoS}_{2}$. ACS Appl. Mater. Interfaces 6, 314-318 (2013).

19. Trolle, M. L., Seifert, G. \& Pedersen, T. G. Theory of excitonic second-harmonic generation in monolayer $\mathrm{MoS}_{2}$. Phys. Rev. B 89, 235410 (2014).

20. Clark, D. et al. Strong optical nonlinearity of CVD-grown $\mathrm{MoS}_{2}$ monolayer as probed by wavelength-dependent second-harmonic generation. Phys. Rev. B 90, 121409 (2014).

21. Seyler, K. L. et al. Electrical control of second-harmonic generation in a $\mathrm{WSe}_{2}$ monolayer transistor. Nat. Nanotechnol 10, 407-411 (2015).

22. Kuc, A., Zibouche, N. \& Heine, T. Influence of quantum confinement on the electronic structure of the transition metal sulfide $\mathrm{TS}_{2}$. Phys. Rev. B 83, 245213 (2011).

23. Kadantsev, E. S. \& Hawrylak, P. Electronic structure of a single $\mathrm{MoS}_{2}$ monolayer. Solid State Commun 152, 909-913 (2012).

24. Wang, Q. H., Kalantar-Zadeh, K., Kis, A., Coleman, J. N. \& Strano, M. S. Electronics and optoelectronics of two-dimensional transition metal dichalcogenides. Nat. Nanotechnol 7, 699-712 (2012).

25. Shi, H., Pan, H., Zhang, Y.-W. \& Yakobson, B. I. Quasiparticle band structures and optical properties of strained monolayer $\mathrm{MoS}_{2}$ and $\mathrm{WS}_{2}$. Phys. Rev. B 87, 155304 (2013).

26. Zahid, F., Liu, L., Zhu, Y., Wang, J. \& Guo, H. A generic tight-binding model for monolayer, bilayer and bulk $\mathrm{MoS}_{2}$. AIP Adv. 3, 052111 (2013).

27. Kormányos, A. et al. Monolayer $\mathrm{MoS}_{2}$ : trigonal warping, the $\gamma$ valley, and spin-orbit coupling effects. Phys. Rev. B 88, 045416 (2013).

28. Qiu, D. Y., Felipe, H. \& Louie, S. G. Optical spectrum of $\mathrm{MoS}_{2}$ : many-body effects and diversity of exciton states. Phys. Rev. Lett. 111, 216805 (2013).

29. Gibertini, M., Pellegrino, F. M., Marzari, N. \& Polini, M. Spin-resolved optical conductivity of two-dimensional group-VIb transition-metal dichalcogenides. Phys. Rev. B 90, 245411 (2014).

30. Margulis, V. A., Muryumin, E. E. \& Gaiduk, E. A. Optical second-harmonic generation from two-dimensional hexagonal crystals with broken space inversion symmetry. J. Phys. Condens. Matter 25, 195302 (2013).

31. Wu, S. et al. Quantum-enhanced tunable second-order optical nonlinearity in bilayer graphene. Nano Lett. 12, 2032-2036 (2012).

32. Brun, S. J. \& Pedersen, T. G. Intense and tunable second-harmonic generation in biased bilayer graphene. Phys. Rev. B 91, 205405 (2015).

33. Hipolito, F., Pedersen, T. G. \& Pereira, V. M. Nonlinear photocurrents in two-dimensional systems based on graphene and boron nitride. Phys. Rev. B 94, 045434 (2016)

34. Xiao, D., Liu, G.-B., Feng, W., Xu, X. \& Yao, W. Coupled spin and valley physics in monolayers of $\mathrm{MoX}_{2}$ and other group-vi dichalcogenides. Phys. Rev. Lett. 108, 196802 (2012).

35. Rostami, H., Roldán, R., Cappelluti, E., Asgari, R. \& Guinea, F. Theory of strain in single-layer transition metal dichalcogenides. Phys. Rev. B 92, 195402 (2015).

36. Rostami, H., Asgari, R. \& Guinea, F. Edge modes in zigzag and armchair ribbons of monolayer $\mathrm{MoS}_{2}$. J. Phys. Condens. Matter 28, 495001 (2016).

37. Rostami, H., Moghaddam, A. G. \& Asgari, R. Effective lattice hamiltonian for monolayer $\mathrm{MoS}_{2}$ : tailoring electronic structure with perpendicular electric and magnetic fields. Phys. Rev. B 88, 085440 (2013).

38. Alidoust, $\mathrm{N}$. et al. Observation of monolayer valence band spin-orbit effect and induced quantum well states in $\mathrm{MoX}_{2}$. Nat. Commun 5, 4673 (2014).

39. Rostami, H. \& Polini, M. Theory of third-harmonic generation in graphene: a diagrammatic approach. Phys. Rev. B 93, 161411 (2016).
40. Bonaccorso, F. et al. Production and processing of graphene and $2 \mathrm{~d}$ crystals. Mater. Today 15, 564-589 (2012).

41. Sundaram, R. et al. Electroluminescence in single layer $\mathrm{MoS}_{2}$. Nano Lett. 13, 1416-1421 (2013).

42. Casiraghi, C. et al. Rayleigh imaging of graphene and graphene layers. Nano Lett. 7, 2711-7 (2007).

43. Zhang, X. et al. Raman spectroscopy of shear and layer breathing modes in multilayer $\mathrm{MoS}_{2}$. Phys. Rev. B 87, 115413 (2013).

44. Säynätjoki, A. et al. Rapid large-area multiphoton microscopy for characterization of graphene. ACS Nano 7, 8441-8446 (2013).

45. Kieu, K., Jones, R. J. \& Peyghambarian, N. Generation of few-cycle pulses from an amplified carbon nanotube mode-locked fiber laser system. IEEE Photon. Technol. Lett. 22, 1521-1523 (2010).

46. Kieu, K., Jones, R. J. \& Peyghambarian, N. High power femtosecond source near 1 micron based on an all-fiber er-doped mode-locked laser. Opt. Express 18, 21350-21355 (2010).

47. Miller, R. C. Optical second harmonic generation in piezoelectric crystals. Appl. Phys. Lett. 5, 17-19 (1964).

48. Chin, A. H., Calderón, O. G. \& Kono, J. Extreme midinfrared nonlinear optics in semiconductors. Phys. Rev. Lett. 86, 3292 (2001).

49. Woodward, R. I. et al. Characterization of the second- and third-order nonlinear optical susceptibilities of monolayer $\mathrm{MoS}_{2}$ using multiphoton microscopy. 2D Mater 4, 011006 (2017).

50. Säynätjoki, A. et al. Ultra-strong nonlinear optical processes and trigonal warping in $\mathrm{MoS}_{2}$ layers. Preprint at https://arxiv.org/abs/1608.04101 (2016).

51. Clark, D. et al. Near bandgap second-order nonlinear optical characteristics of $\mathrm{MoS}_{2}$ monolayer transferred on transparent substrates. Appl. Phys. Lett. 107, 131113 (2015).

52. Le, C. T. et al. Impact of selenium doping on resonant second harmonic generation in monolayer $\mathrm{MoS}_{2}$. ACS Photon. 4, 38-44 (2017).

53. Liu, $\mathrm{H}$. et al. High-harmonic generation from an atomically thin semiconductor. Nat. Phys 13, 262-265 (2017).

54. Janisch, C. et al. Extraordinary second harmonic generation in tungsten disulfide monolayers. Sci. Rep. 4, 5530 (2014).

55. Le, C. T. et al. Nonlinear optical characteristics of monolayer MoSe 2 . Ann. Phys 528, 551-559 (2016)

56. Kumar, N. et al. Third harmonic generation in graphene and few-layer graphite films. Phys. Rev. B 87, 121406 (2013).

57. Hendry, E., Hale, P. J., Moger, J., Savchenko, A. \& Mikhailov, S. Coherent nonlinear optical response of graphene. Phys. Rev. Lett. 105, 097401 (2010)

58. Cheng, J., Vermeulen, N. \& Sipe, J. Third order optical nonlinearity of graphene. New J. Phys. 16, 053014 (2014).

59. Hong, S.-Y. et al. Optical third-harmonic generation in graphene. Phys. Rev. X 3, 021014 (2013).

60. Neto, A. H. C., Guinea, F., Peres, N. M., Novoselov, K. S. \& Geim, A. K. The electronic properties of graphene. Rev. Mod. Phys. 81, 109 (2009).

61. Nair, R. R. et al. Fine structure constant defines visual transparency of graphene. Science 320, 1308-1308 (2008).

62. Ramasubramaniam, A. Large excitonic effects in monolayers of molybdenum and tungsten dichalcogenides. Phys. Rev. B 86, 115409 (2012).

63. Cheiwchanchamnangij, T. \& Lambrecht, W. R. L. Quasiparticle band structure calculation of monolayer, bilayer, and bulk $\mathrm{MoS}_{2}$. Phys. Rev. B 85, 205302 (2012).

64. Mak, K. F. et al. Tightly bound trions in monolayer $\mathrm{MoS}_{2}$. Nat. Mater. 12, 207-211 (2013)

65. Grüning, M. \& Attaccalite, C. Second harmonic generation in h-BN and $\mathrm{MoS}_{2}$ monolayers: role of electron-hole interaction. Phys. Rev. B 89, 081102 (2014).

66. Wang, G. et al. Giant enhancement of the optical second-harmonic emission of $\mathrm{WSe}_{2}$ monolayers by laser excitation at exciton resonances. Phys. Rev. Lett. 114, 097403 (2015).

67. Haug, H. \& Koch, S. W. Quantum Theory of the Optical and Electronic Properties of Semiconductors (World Scientific Publishing, 2009).

68. Zhu, S.-n, Zhu, Y.-y \& Ming, N.-b Quasi-phase-matched third-harmonic generation in a quasi-periodic optical superlattice. Science 278, 843-846 (1997).

69. Butcher, P. N. \& Cotter, D. The Elements of Nonlinear Optics (Cambrige University Press, 1990).

70. Radisavljevic, B., Radenovic, A., Brivio, J., Giacometti, I. V. \& Kis, A. Singlelayer $\mathrm{MoS}_{2}$ transistors. Nat. Nanotechnol 6, 147-150 (2011).

\section{Acknowledgements}

We thank M.J. Huttunen and R.W. Boyd for useful discussions. We acknowledge funding from the Academy of Finland (Nos: 276376, 284548, 295777, 298297, and 304666), TEKES (NP-Nano, OPEC), Royal Academy of Engineering (RAEng) Research Fellowships, Fondazione Istituto Italiano di Tecnologia, the Graphene Flagship, ERC grants Hetero2D, Nokia Foundation, EPSRC Grants EP/K01711X/1, EP/K017144/1, EP/ 
L016087/1, AFOSR COMAS MURI (FA9550-10-1-0558), ONR NECom MURI, CIAN NSF ERC under Grant EEC-0812072, and TRIF Photonics funding from the state of Arizona and the Micronova, Nanofabrication Centre of Aalto University.

\section{Author contributions}

A.S., L.K., A.A., S.M., K.K., and Z.S. carried out the multiphoton experiments and data analysis. Samples preparation and characterization were carried out by A.L. and A.C.F. The nonlinear response function calculations were carried out by H.R. and M.P. Sample fabrication, multiphoton characterization, and analysis were coordinated by R.A.N., N.P., H.L., A.C.F., M.P., and Z.S. All authors contributed to the manuscript.

\section{Additional information}

Supplementary Information accompanies this paper at 10.1038/s41467-017-00749-4.

Competing interests: The authors declare no competing financial interests.

Reprints and permission information is available online at http://npg.nature.com/ reprintsandpermissions/
Publisher's note: Springer Nature remains neutral with regard to jurisdictional claims in published maps and institutional affiliations.

(c) (i) Open Access This article is licensed under a Creative Commons Attribution 4.0 International License, which permits use, sharing, adaptation, distribution and reproduction in any medium or format, as long as you give appropriate credit to the original author(s) and the source, provide a link to the Creative Commons license, and indicate if changes were made. The images or other third party material in this article are included in the article's Creative Commons license, unless indicated otherwise in a credit line to the material. If material is not included in the article's Creative Commons license and your intended use is not permitted by statutory regulation or exceeds the permitted use, you will need to obtain permission directly from the copyright holder. To view a copy of this license, visit http://creativecommons.org/ licenses/by/4.0/.

(C) The Author(s) 2017 